\title{
Rhizoma Coptidis: A Potential Cardiovascular Protective Agent
}

\author{
Hui-Li Tan ${ }^{1,2}$, Kok-Gan Chan ${ }^{3}$, Priyia Pusparajah ${ }^{2}$, Acharaporn Duangjai ${ }^{4,5}$, \\ Surasak Saokaew ${ }^{1,4,6}$, Tahir Mehmood Khan ${ }^{1,7}$, Learn-Han Lee ${ }^{1,4 *}$ and Bey-Hing Goh ${ }^{1,4 *}$ \\ ${ }^{1}$ Novel Bacteria and Drug Discovery Research Group, School of Pharmacy, Monash University Malaysia, Bandar Sunway, \\ Malaysia, ${ }^{2}$ Biomedical Research Laboratory, Jeffrey Cheah School of Medicine and Health Sciences, Monash University \\ Malaysia, Bandar Sunway, Malaysia, ${ }^{3}$ Division of Genetic and Molecular Biology, Faculty of Science, Institute of Biological \\ Sciences, University of Malaya, Kuala Lumpur, Malaysia, ${ }^{4}$ Center of Health Outcomes Research and Therapeutic Safety, \\ School of Pharmaceutical Sciences, University of Phayao, Phayao, Thailand, ${ }^{5}$ Division of Physiology, School of Medical \\ Sciences, University of Phayao, Phayao, Thailand, ${ }^{6}$ Faculty of Pharmaceutical Sciences, Pharmaceutical Outcomes \\ Research Center, Naresuan University, Phitsanulok, Thailand, ${ }^{7}$ Department of Pharmacy, Abasyn University Peshawar, \\ Peshawar, Pakistan
}

\section{OPEN ACCESS}

Edited by:

Lyndy Joy McGaw,

University of Pretoria, South Africa

Reviewed by:

Wenwu Liu,

Second Military Medical University,

China

Adeyemi Oladapo Aremu,

University of KwaZulu-Natal,

South Africa

*Correspondence:

Learn-Han Lee

lee.learn.han@monash.edu

Bey-Hing Goh

goh.bey.hing@monash.edu

Specialty section:

This article was submitted to

Ethnopharmacology,

a section of the journal

Frontiers in Pharmacology

Received: 04 August 2016 Accepted: 20 September 2016

Published: 07 October 2016

Citation:

Tan H-L, Chan K-G, Pusparajah P,

Duangjai A, Saokaew S, Mehmood

Khan T, Lee L-H and Goh B-H (2016)

Rhizoma Coptidis: A Potential

Cardiovascular Protective Agent.

Front. Pharmacol. 7:362.

doi: 10.3389/fphar.2016.00362
Cardiovascular diseases (CVDs) are among the leading causes of morbidity and mortality in both the developed and developing world. Rhizoma coptidis (RC), known as Huang Lian in China, is the dried rhizome of medicinal plants from the family Ranunculaceae, such as Coptis chinensis Franch, C. deltoidea C.Y. Cheng et Hsiao, and C. teeta Wall which has been used by Chinese medicinal physicians for more than 2000 years. In China, RC is a common component in traditional medicines used to treat CVD associated problems including obesity, diabetes mellitus, hyperlipidemia, hyperglycemia and disorders of lipid metabolism. In recent years, numerous scientific studies have sought to investigate the biological properties of RC to provide scientific evidence for its traditional medical uses. RC has been found to exert significant beneficial effects on major risk factors for CVDs including anti-atherosclerotic effect, lipid-lowering effect, antiobesity effect and anti-hepatic steatosis effect. It also has myocardioprotective effect as it provides protection from myocardial ischemia-reperfusion injury. These properties have been attributed to the presence of bioactive compounds contained in RC such as berberine, coptisine, palmatine, epiberberine, jatrorrhizine, and magnoflorine; all of which have been demonstrated to have cardioprotective effects on the various parameters contributing to the occurrence of CVD through a variety of pathways. The evidence available in the published literature indicates that $R C$ is a herb with tremendous potential to reduce the risks of CVDs, and this review aims to summarize the cardioprotective properties of $\mathrm{RC}$ with reference to the published literature which overall indicates that $\mathrm{RC}$ is a herb with remarkable potential to reduce the risks and damage caused by CVDs.

Keywords: coptis root, Huang Lian, Coptis chinensis Franch, cardiovascular diseases, ethnopharmacology

\section{INTRODUCTION}

Cardiovascular diseases (CVDs) appears set to continue as the largest cause of death and disease burden across the globe. They include a wide spectrum of life-threatening disorders such as coronary heart disease (CHD), cerebrovascular disease and peripheral arterial disease, all of which result from impairment to the heart and blood vessels (Wallace, 2011). Among the risk factors 
strongly associated with these disorders are high levels of lowdensity lipoprotein (LDL) cholesterol, hypertension, diabetes, and abdominal obesity (Walden and Tomlinson, 2011).

Rhizoma coptidis (RC), known as Huang Lian in China, is the dried rhizome of medicinal plants from the family Ranunculaceae, including Coptis chinensis Franch, C. deltoidea C.Y. Cheng et Hsiao, and C. teeta Wall (Chen et al., 2008; Ma et al., 2012). It is a well-known herb in traditional Chinese medicine and has a long history with its pharmacological uses first mentioned in the Shen Nong Ben Cao Jing (a compilation of information regarding Chinese herbs dating back to $2800 \mathrm{BC}$ ) in the Eastern Han Dynasty (Yi et al., 2013). Ancient beliefs state that it is "cold" in nature and is able to remove damp heat, fire or toxicity (Wang et al., 2014). For over 2000 years, Chinese medicinal physicians have used RC as a food additive and herbal medicine for its antibacterial, antiviral, anti-inflammatory, antihyperglycemic and hypolipidemic activities (Kou et al., 2016). Today, RC is still widely utilized in herbal medicine for the treatment of various conditions. This is evident based on a survey of patented medicines in China which reveals that RC is commonly used as one of the ingredients in preparations to treat obesity, diabetes mellitus, hyperlipidemia, hyperglycemia and lipid metabolism disorders (Chen, 2009; Ye et al., 2009; Guo, 2012; Li et al., 2015; Wang, 2015). Given the potential benefits in seeking new approaches to treating and preventing CVDs, there has been tremendous interest among the scientific community in exploring the biological properties of RC and providing scientific evidence for its traditional medical uses. At the same time, it is also crucial to investigate the phytoconstituents that are

\footnotetext{
Abbreviations: ACC, acetyl coenzyme A carboxylase; ADP, adenosine monophosphate; AMPK, adenosine 5'-monophosphate (AMP)-activated protein kinase; AP-1, activator protein 1; ASBT, apical sodium-dependent bile salt transporter; ATP, adenosine triphosphate; $\mathrm{Bax}, \mathrm{Bcl}-2$-associated $\mathrm{X}$ protein; $\mathrm{Bcl}-2$, B cell lymphoma 2; BNIP3, BCL2/adenovirus E1B 19kDa interacting protein 3; C/EBP- $\alpha$, CCAAT/enhancer-binding protein- $\alpha$; CK, creatine kinase; COX-2, cyclooxygenase-2; CPT1 $\alpha$, carnitine palmitoyl transferase 1-alpha; cTn1, cardiac troponin I; CYP7A1, cholesterol 7-alpha-hydroxylase; EMMPRIN, extracellular matrix metalloproteinase inducer; eNOS, endothelial nitric oxide synthase; FAS, fatty acid synthase; FXR, farnesoid X receptor; GSK $3 \beta$, glycogen synthase kinase $3 \beta$; HDL, high density lipoprotein; HDL-c, high density lipoprotein cholesterol; Hes1, Hairy and enhancer of split 1; HMG-CoA, 3-hydroxy3methylglutary-CoA; HMGCR, 3-Hydroxy-3-methylglutaryl CoA reductase; HO-1, heme-oxygenase-1; HUVECs, human umbilical vein endothelial cells; IL, Interleukin; I/R, ischemia/reperfusion; iNOS, inducible nitric oxide synthase; JAK, Janus kinase; JNK, c-Jun N-terminal kinase; LDH, lactate dehydrogenase; LDL, low density lipoprotein; LDL-c, low density lipoprotein cholesterol; LDLR, low-density lipoprotein receptor; LPS, Lipopolysaccharide; LXR, liver X receptor; MAPK, p38 mitogen-activated protein kinase; MCP-1, monocyte chemoattractant protein-1; MDA, malondialdehyde; MMP, matrix metalloproteinase; mTORC2, mammalian target of rapamycin complex 1; NFкB, Nuclear factor-kappaB; Ox-LDL, oxidized low density lipoprotein; pACC, phosphorylated-ACC; PGC1 $\alpha$, peroxisome proliferator-activated receptor gamma coactivator 1-alpha; PI3K, phosphoinositide 3-kinase; PMA, phorbol 12-myristate 13-acetate; PPAR, proliferator-activated receptor; PTEN, Phosphatase and tensin homolog deleted on chromosome 10; ROCK, Rho-kinase; ROS, reactive oxygen species; SIRT1, Sirtuin 1; SOD, superoxide dismutase; SREBP2, Sterol regulatory element binding transcription factor 2; STAT3, signal transducer and activator of transcription 3; TBA, total bile acids; TBARS, thiobarbituric acid reactive substances; TC, total cholesterol; TG, triglyceride; TGR5, G-protein coupled bile acid receptor; TLR4, toll-like receptor 4; TNF, tumor necrosis factor; TXNIP, thioredoxin-interacting protein; UCP 2, uncoupling protein 2; VMSCs, vascular smooth muscle cells.
}

responsible for the biological properties (Moghadamtousi et al., 2013; Tan et al., 2015). Based on current knowledge, the major bioactive compounds contributing to RC's bioactive properties are berberine, coptisine, palmatine, epiberberine, jatorrhizine and magnoflorine, as illustrated in Figure 1 (Hung et al., 2007a; Kou et al., 2016). There is a large of body of work suggesting that $\mathrm{RC}$ has protective properties against several major risk factors and damage caused by CVDs. This review aims to summarize the currently available evidence of RC's cardioprotective propertiesboth in vitro and in vivo studies were included and are summarized in Table 1.

\section{CARDIOPROTECTIVE ACTIVITIES OF RHIZOMA COPTIDIS}

\section{Anti-Atherosclerotic Effect}

Atherosclerosis is one of the most important causative factors of certain CVDs such as myocardial infarction (MI), heart failure, stroke and claudication (Frostegård, 2013). Atherosclerosis refers to a specific form of arteriosclerosis in which an artery-wall thickens due to invasion and accumulation of lipid laden white blood cells (foam cells) and proliferation of intimalsmooth-muscle cell forming a fibrofatty plaque (Lucas and Greaves, 2001). There have also been studies supporting the anti-atherosclerotic effects of RC. For example, a study on the anti-atherosclerotic potential of Ger-Gen-Chyn-Lian-Tang (a traditional mixture which contains berberine as one of its major active components) has demonstrated reduced atherosclerotic lesions and collagen expression within the atheroma plaques in mice. In addition, in vitro work has also shown a reduction in migration of vascular smooth muscle cells, which is significant as pathological migration of these cells represents a key step in the pathogenesis of atherosclerosis (Ho et al., 2012).

Reactive oxygen species (ROS) play a crucial role in the development and progression of atherosclerotic lesions because the formations of lesions are related to several ROS-regulated events. It was found that uncoupling protein 2 (UCP-2), the mitochondrial inner membrane protein can provide antioxidant mediated defense by negatively regulating ROS production (Moukdar et al., 2009). Utilizing a mouse model, Wang et al. (2011) reported that chronic administration of berberine resulted in significant reduction of aortic lesions, diminished oxidative stress and lowered expression of adhesion molecules in the aorta; this anti-atherogenic activity was due to AMPK-dependent ROS suppression through enhanced expression of UCP-2. Oxidative modification of LDL is also believed to be one of the main risk factors leading to atherosclerosis (Itabe, 2009); berberine-one of the major bioactive components of $\mathrm{RC}$-has been reported to inhibit oxidation of LDL by virtue of its significant antioxidant activity (Hsieh et al., 2007). Berberine also appears to protect against oxidized-LDL induced apoptosis as demonstrated by reduced levels of mitochondrial cytochrome $C$, cleaved apoptotic effectors, caspase 3 and poly(ADP-ribose) polymerase in human umbilical vein endothelial cells treated with this compound (Hsieh et al., 2007). Magnoflorine, another alkaloid isolated from RC extract also possesses similarly beneficial effects; an effective antioxidant, it prevents the oxidation of various forms of LDL, 


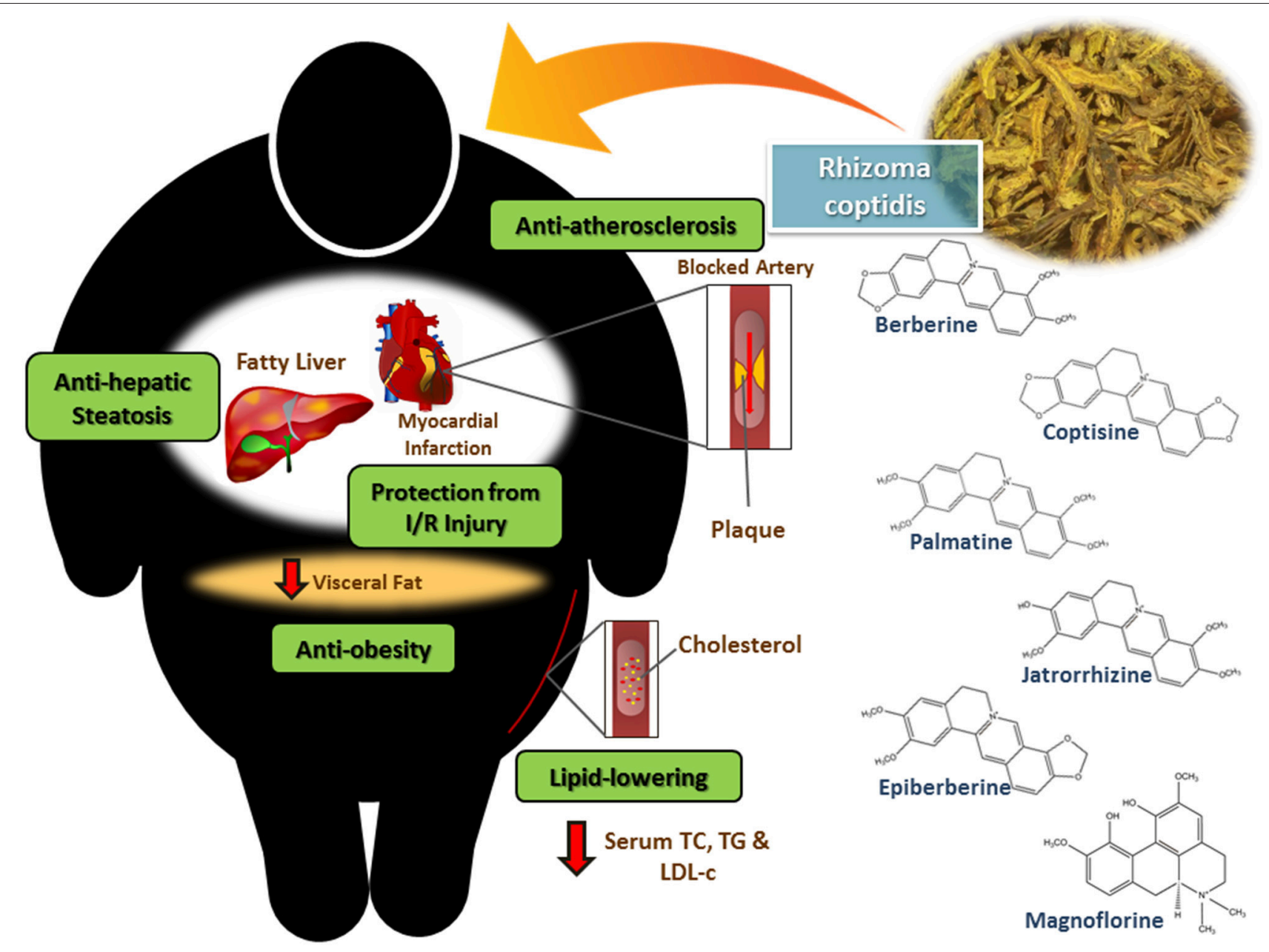

FIGURE 1 | Rhizoma coptidis which contains alkaloids such as berberine, coptisine, palmatine, epiberberine, jatrorrhizine and magnoflorine exerts cardioprotective activity through its anti-atherosclerotic effect, protection from myocardial ischemia-reperfusion injury, lipid-lowering effect, anti-obesity effect and anti-hepatic steatosis effect.

including native, glycated and glycoxidated LDL (Hung et al., 2007a). Aside from this, magnoflorine also significantly inhibits $\mathrm{Cu}^{2+}$ and thermo-labile radical initiator (AAPH)-induced lipid peroxidation of high density lipoprotein (HDL); thus resulting in HDL retaining its ability to protect LDL from oxidation (Hung et al., 2007b).

Chronic inflammatory processes are a key additional factor leading to the development of atherosclerotic lesions in the vessel wall (Remppis et al., 2010). Hence, substances with anti-inflammatory property may have the potential to prevent atherosclerosis. Remppis et al. (2010) studied the effect of $\mathrm{RC}$ extract and berberine on lipopolysaccharide (LPS)-induced inflammatory activity in a murine macrophage cell line. The results of this study demonstrated that RC extract and berberine inhibit the production of monocyte chemoattractant protein 1 (MCP-1/CCL2) in macrophages, likely via inhibition of the transcription factors activator protein 1 (AP-1) and nuclear factor-kappaB (NFкB). Coptisine, another of the bioactive compounds in RC, also possesses demonstrable antiinflammatory property. It resulted in decreased nitric oxide (NO) production in LPS-stimulated macrophages via inhibited protein and mRNA expression of inducible nitro oxide synthase (iNOS); additionally, the expression of pro-inflammatory cytokines interleukin (IL)- $1 \beta$ and IL- 6 was inhibited at transcriptional level. The proposed underlying mechanism for these observations was blockade of $\mathrm{NF \kappa B}$, mitogen-activated protein kinases (MAPK), and phosphoinositide-3-kinase (PI3K)/Akt activation, which are the intracellular inflammation signaling pathways in macrophages (Supriady et al., 2015; Wu et al., 2016).

The accumulation of lipid-laden foam cells is also an important step in the progression of atherosclerosis (Chi et al., 2014). The authors demonstrated that berberine effectively inhibited oxidized-LDL-induced foam cell formation through the activation of adenosine $5^{\prime}$-monophosphate-activated protein kinase (AMPK)-sirtuin 1-peroxisome proliferator-activated receptor gamma (PPAR- $\gamma$ ) pathway as well as through reduced 


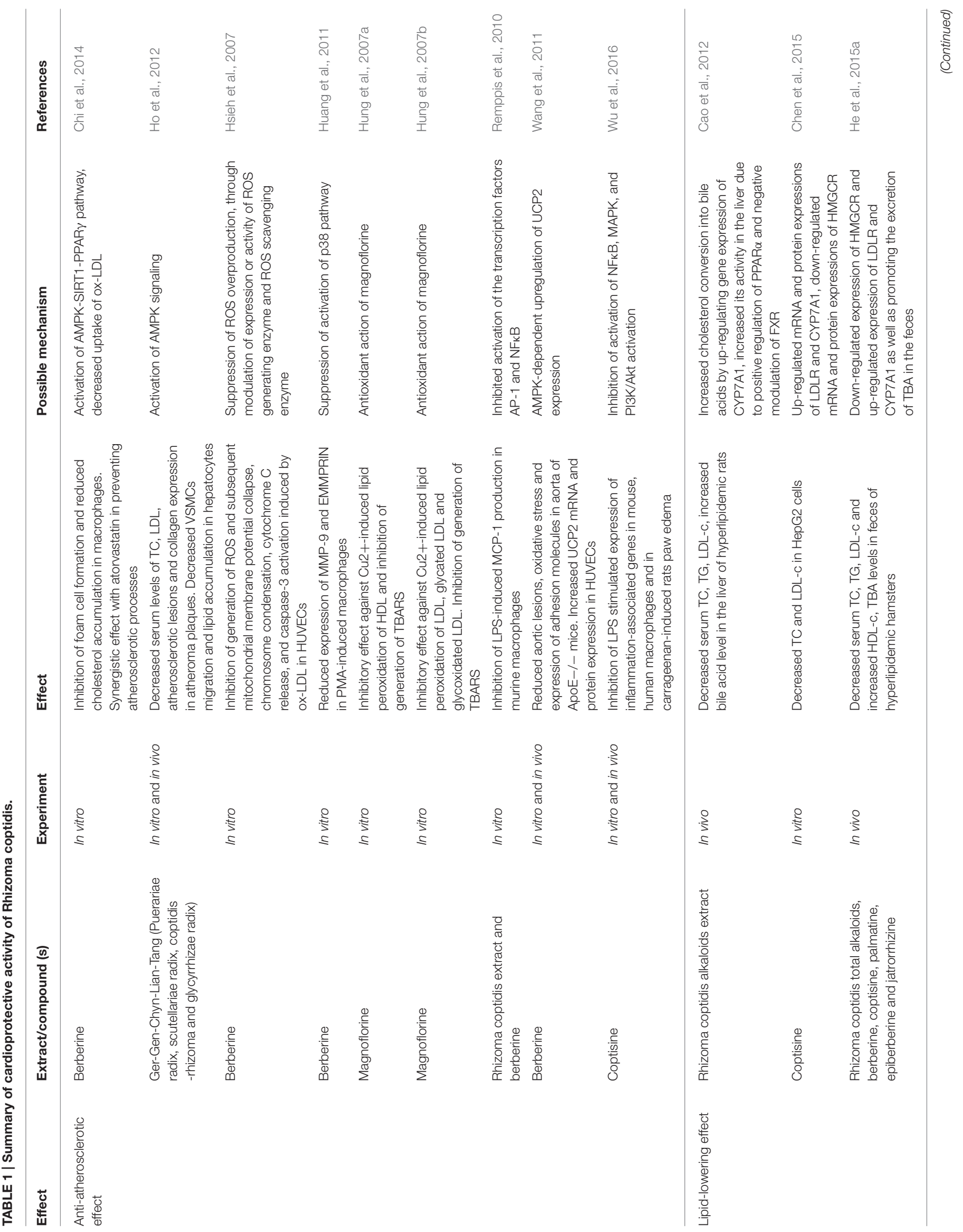




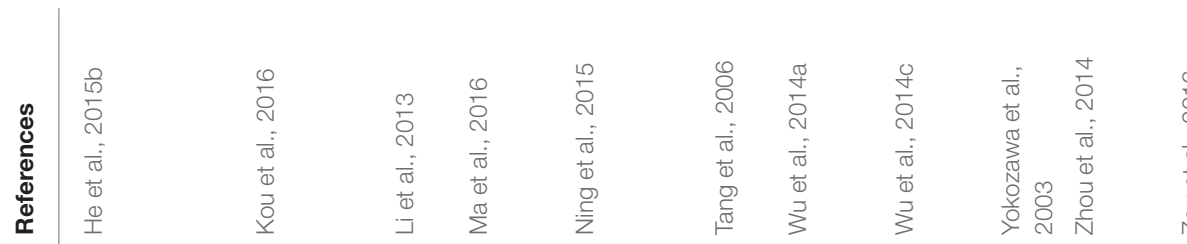
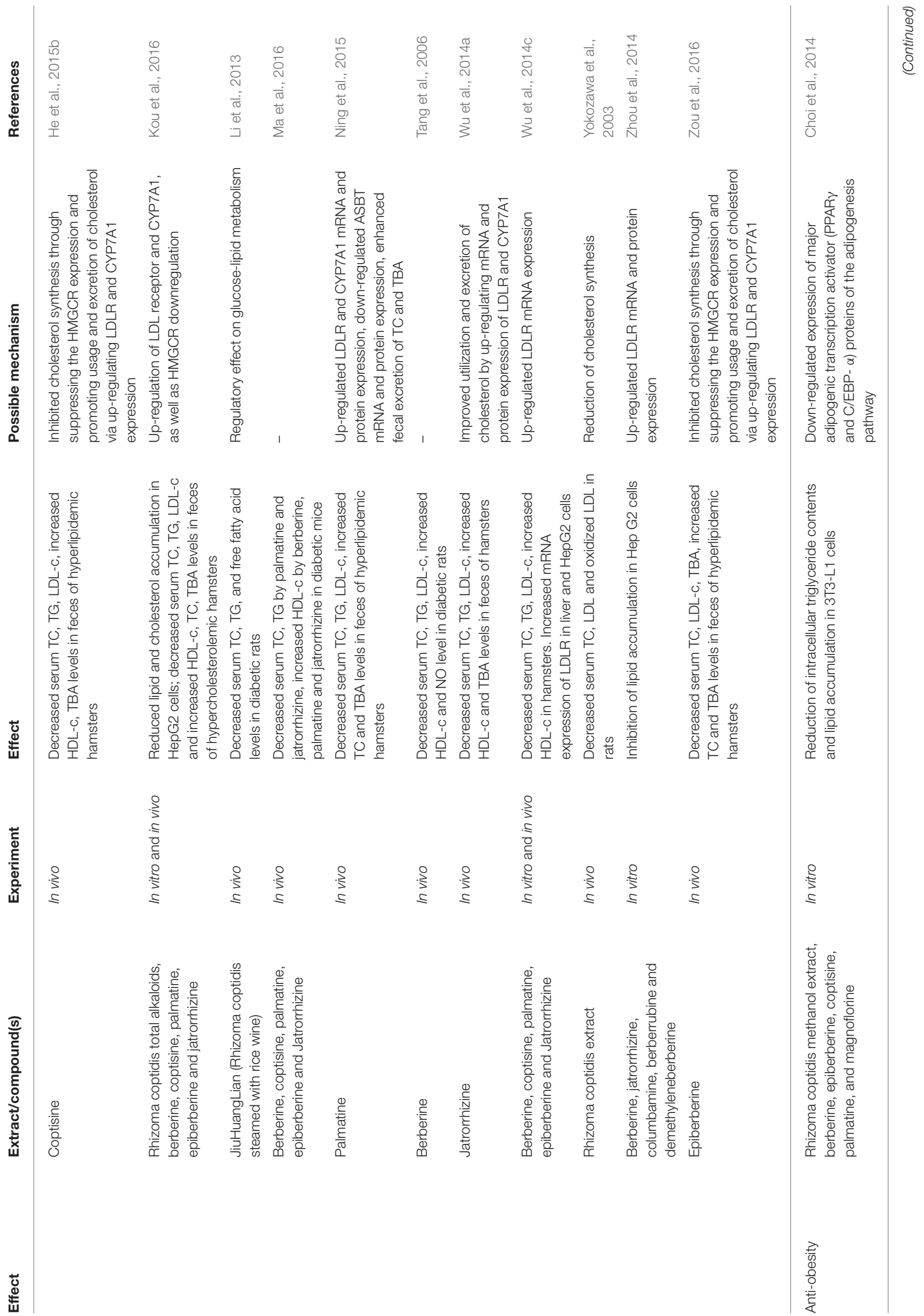


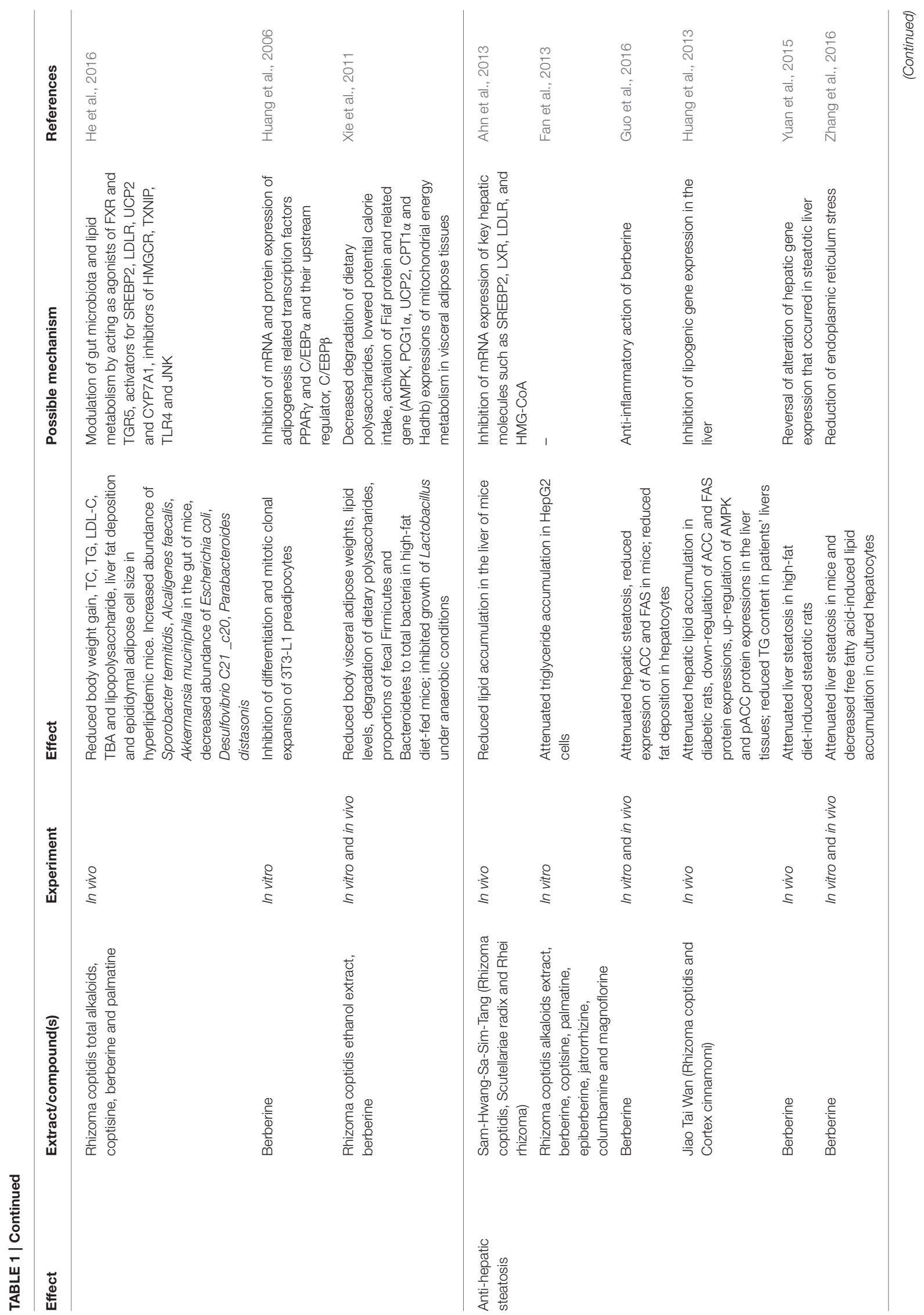




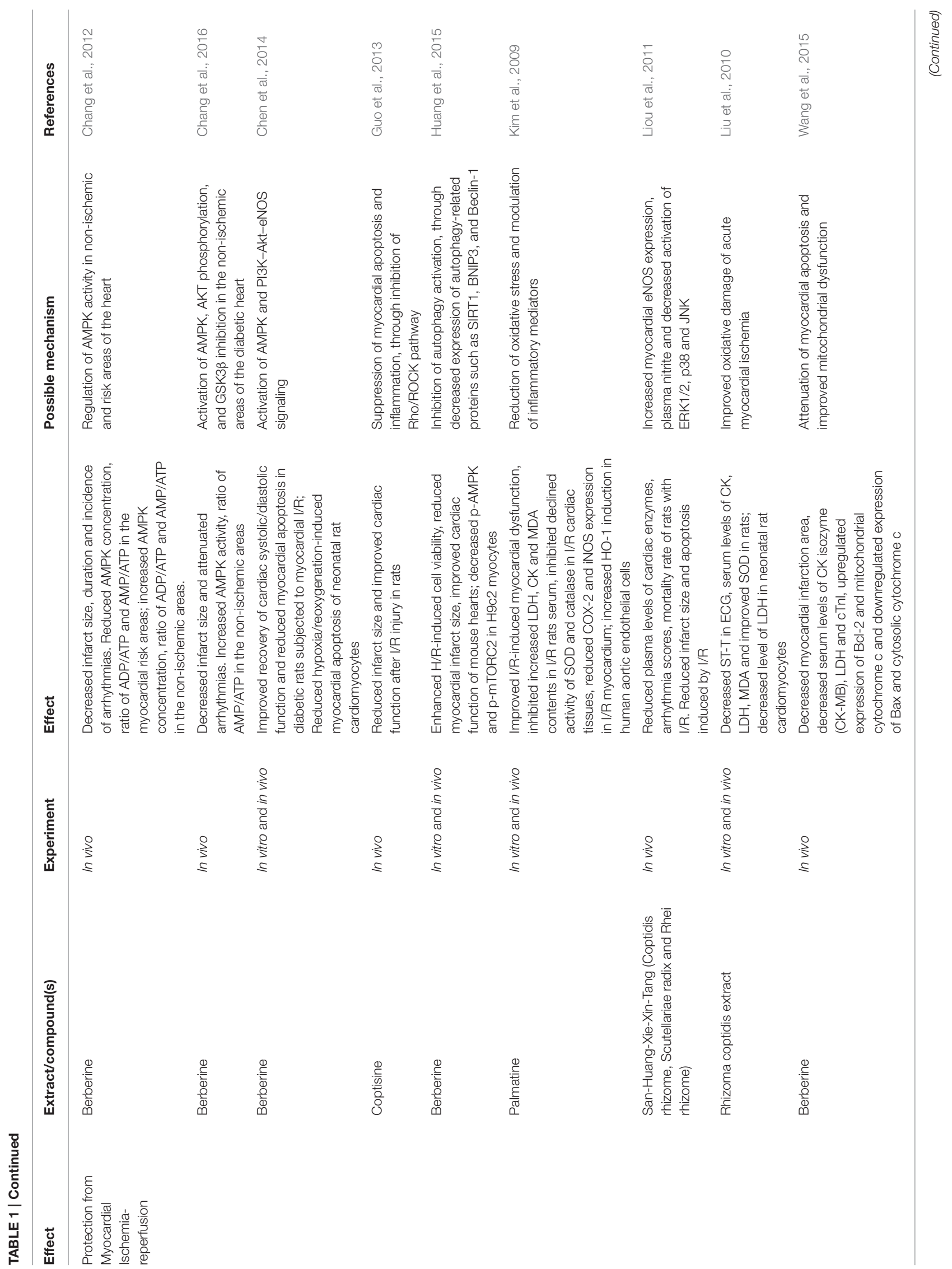


uptake of oxidized LDL by foam cells. Furthermore, the same study showed that combination therapy of berberine and atorvastatin (a well-established anti-atherosclerotic drug) was more effective in preventing atherosclerotic processes compared to administration of atorvastatin alone.

Clinically, the major concern when artherosclerosis is present is the occurrence of plaque disruption which may then lead to unstable angina, or an outright MI. Plaque progression and destabilization is due to the overexpression of matrix metalloproteinases-9 (MMP-9) and extracellular matrix metalloproteinase inducer (EMMPRIN) in monocytes/macrophages (Cao et al., 2014). Berberine inhibits the up-regulation of MMP-9 and EMMPRIN in phorbol 12myristate 13-acetate-induced macrophages by suppressing the activation of p38 pathway, showing its ability to stabilize atherosclerotic plaque (Huang et al., 2011).

RC seems to have multiple active components that target varying stages of the development of atherosclerosis through a variety of pathways, thus making it an effective antiatherosclerotic agent.

\section{Lipid-Lowering Effect}

Hyperlipidemia or dyslipidemia, which is characterized by increased plasma triglyceride concentration and lowered HDL cholesterol, is one of the major risk factor contributing to the progression of CVDs such as CHD and peripheral artery disease. Hence, maintenance of total cholesterol (TC) level within the normal range can be an effective strategy to lower the risk of cardiovascular events (Zou et al., 2016). The liver is the main site of cholesterol homeostasis maintenance, which involves several distinct processes including biosynthesis via the activity of 3-hydroxy-3-methylglutaryl coenzyme A reductase (HMGCR), uptake by low density lipoprotein receptors (LDLR), release of lipoprotein in the blood, storage, degradation and conversion into bile acids (Trapani et al., 2012). RC possesses lipid-lowering effects as shown by the lowered cholesterol levels in different animal models treated with RC extract (Yokozawa et al., 2003; Cao et al., 2012; Kou et al., 2016). Studies have indicated that the five main bioactive compounds in RC (berberine, coptisine, palmatine, epiberberine, and jatrorrhizine) exert their lipid-lowering effects through distinct mechanisms. Berberine's hypolipidemic effect was the result of up-regulation of LDLR mRNA and protein expression (Zhou et al., 2014). Coptisine treatment also resulted in increased expression of LDLR, and additionally was associated with up-regulated expression of cholesterol 7-alpha-hydroxylase (CYP7A1) and down-regulated expression of HMGCR (Chen et al., 2015; He et al., 2015b). In addition to up-regulated expression of LDLR and CYP7A1, treatment with palmatine and epiberberine also caused downregulation of apical sodium-dependent bile salt transporter (ASBT) mRNA and protein expression, as well as enhanced fecal excretion of TC and total bile acids (Ning et al., 2015; Zou et al., 2016). Jatrorrhizine has been linked to up-regulated mRNA and protein expression of LDLR and CYP7A1 but it has no effect on the expression of ASBT and HMGCR (Wu et al., 2014a). Comparative studies have been done on the lipidlowering effect of individual alkaloids and among those tested, 
coptisine was reported to have the highest lipid-lowering effect (Wu et al., 2014c). More recently, research involving combined treatment with the alkaloids in RC extract has also been carried out. While each of the compounds administered in isolation did demonstrate lipid-lowering effect, the administration of a combination of these alkaloids resulted in synergistic effects, with combination therapy resulting in a significantly greater lipidlowering effect compared to treatment with a single alkaloid. The main mechanisms associated with this effect are thought to be the retardation of cholesterol synthesis and accelerated clearance of cholesterol (He et al., 2015a; Kou et al., 2016).

Type 2 diabetes is strongly associated with significant morbidity and mortality due to cardiovascular complications. This is in part due to the impaired utilization of carbohydrate that is part of the metabolic profile of diabetes, which results in accelerated lipolysis and thus hyperlipidemia or dyslipidemia (Vijayaraghavan, 2010; Ma et al., 2016). RC has been shown to have the ability to attenuate the impaired lipid metabolism associated with diabetes. JiuHuangLian (RC steamed with rice wine), a traditional Chinese medicine preparation has been reported to restore the disordered lipid metabolism of type 2 diabetic rats, by decreasing the TC, total glyceride and free fatty acid levels (Li et al., 2013). The anti-hyperlipidemic effect of the alkaloids isolated from RC have been further tested on diabetic mice. Palmatine and jatrorrhizine were shown to decrease the concentrations of serum TC and triglyceride; while elevated HDL cholesterol levels were found in diabetic mice administered with palmatine, jatrorrhizine and berberine (Ma et al., 2016). In another study, there were decreased serum TC, triglyceride, LDL cholesterol and increased HDL cholesterol in diabetic rats treated with berberine (Tang et al., 2006), showing the ability of RC to attenuate the impaired lipid metabolism in diabetic conditions.

In short, RC has remarkable lipid-lowering effects, mainly through inhibition of cholesterol synthesis, in combination with increased usage and excretion of cholesterol.

\section{Anti-Obesity Effect}

Obesity has become a global epidemic and is strongly associated with occurrence of CVDs including heart failure, CHD, sudden cardiac death and atrial fibrillation (Lavie et al., 2009). As the condition is characterized by accumulation of extra body fat into adipocytes, the typical feature of obesity is an increase in the number and size of adipocytes. The etiology of obesity is rooted in the process of differentiation of preadipocytes into adipocytes, known as adipogenesis (Kwak et al., 2013; Choi et al., 2014). The key transcription regulator genes involved in adipogenesis are PPAR $-\gamma$ and CCAAT/enhancer binding protein alpha (C/EBP$\alpha)$. The inhibition of these two transcription factors could represent an effective method of inhibiting adipogenesis (Choi et al., 2014). RC has potential as an anti-obesity agent as the extract of RC as well as its isolated alkaloids have demonstrated the ability to inhibit cellular triglyceride accumulation in 3T3-L1 adipocytes. Significant down-regulation of expression of PPAR$\gamma$ and C/EBP- $\alpha$ by the alkaloids was also demonstrated, which is consistent with the reported mechanism for the anti-adipogenic activity of berberine (Huang et al., 2006).
The anti-obesity potential of RC has also been demonstrated in in vivo studies. The administration of RC and berberine to mice fed a high fat diet has resulted in significantly lower body and visceral adipose weight. Interestingly, this effect has been related to the antimicrobial activities of both $\mathrm{RC}$ and berberine; with reduced fecal microbes resulting in decreased degradation of dietary polysaccharides, lower calorie intake and de novo lipogenesis. This was followed by activation of fasting-induced adipose factor (Fiaf) protein and related gene expressions of mitochondrial energy metabolism such as AMPK, PGC1 $\alpha$, UCP2, CPT1 $\alpha$, and Hadhb in visceral adipose tissue (Xie et al., 2011). Recently, the gut microbiota of obese mice treated with RC alkaloids has been further studied, demonstrating an increased abundance of microbial species negatively associated with obesity namely Sporobacter termitidis, Alcaligenes faecalis, and Akkermansia muciniphila, and a decreased abundance of the microbial species positively associated with obesity namely Escherichia coli, Desulfovibrio C21_c20 and Parabacteroides distasonis. This indicates the ability of $\mathrm{RC}$ to prevent the development of obesity by restoring the balance of disrupted gut microflora associated with the obese condition (He et al., 2016). $\mathrm{RC}$ appears to be effective in preventing obesity as it is able to inhibit adipogenesis and modulate the gut microflora that plays important role for the development of obesity.

\section{Anti-Hepatic Steatosis Effect}

Nonalcoholic fatty liver disease (NAFLD) - a common liver disease with a disease spectrum ranging from simple steatosis to steatohepatitis-is regarded as the hepatic manifestation of metabolic syndrome (Liu and Lu, 2014). Recently, hepatic steatosis has been identified as one of the associated risk factors closely related to the morbidity and mortality of CVDs. It has been reported that patients with NAFLD have higher risk of getting CHD compared to the general population (Pisto et al., 2014). There are several possible mechanisms by which hepatic steatosis may contribute to the risk of CVDs including oxidative stress, inflammation, dyslipidemia, ischemia reperfusion injury, visceral fat, low adiponectin, ectopic adipose tissue distribution, endothelial dysfunction and postprandial dyslipidemia (Liu and $\mathrm{Lu}, 2014)$. In spite of the many advances in modern medicine, there is as yet no effective treatment of NAFLD (Huang et al., 2013). Herbal medicine has been an area of interest for scientists seeking new modalities of treatment of hepatic steatosis due to the low incidence of side effects and promising therapeutic benefits (Xiao et al., 2013; Tan et al., 2016). For example, Jiao Tai Wan, a classic traditional Chinese prescription consisting of RC and Cortex Cinamomi was able to inhibit hepatic lipid accumulation in diabetic rats and humans, due to down-regulation of lipogenic gene expression and inhibition of lipogenesis in liver tissue (Huang et al., 2013). Sam-HwangSa-Sim-Tang, a Korean traditional preparation which contains RC, Scutellaria radix and Rhei rhizoma also exhibited protective effect against hepatic steatosis by inhibiting the expression of hepatic molecules that modulate cholesterol metabolism (Ahn et al., 2013). While RC extract clearly does have significant potential as an anti-hepatic steatosis agent, there has been interest in identifying which of the extract's active components exert 
the greatest anti-hepatic steatosis effect. Using a free fatty-acid induced hepatic steatosis HepG2 cell assay, Fan et al. (2013) demonstrated that berberine and coptisine are the alkaloids with strongest inhibitory effect on triglyceride accumulation. This is consistent with the findings of other studies that proposed berberine as the effective compound against hepatic steatosis (Yuan et al., 2015; Guo et al., 2016; Zhang et al., 2016). In short, $\mathrm{RC}$ possesses anti-hepatic steatosis effect which is related to its ability to inhibit hepatic lipogenesis.

\section{Protection from Myocardial Ischemia-Reperfusion Injury}

Coronary artery disease resulting in compromised oxygen supply to the myocardium is a common cause of morbidity and mortality. Medical intervention now makes it possible to achieve rapid restoration of blood flow to the ischemic myocardium following an acute event, which should produce better clinical outcomes as prompt restoration of blood circulation helps to prevent further tissue injury. However, the restoration of blood flow or reperfusion, will also cause injury, which is known as myocardial ischemia reperfusion injury ( $\mathrm{I} / \mathrm{R}$ ) (Yu et al., 2015). Apoptosis is an important cellular mechanism resulting in $\mathrm{I} / \mathrm{R}$ injury, with oxidative stress representing one of the major contributing factors promoting apoptosis. The excessive production of ROS which are mainly produced by mitochondria will initiate apoptotic signaling (Chan et al., 2012, 2015). Hence, reducing oxidative stress may help inhibit apoptosis thus potentially providing an effective strategy to attenuate I/R injury (Fan et al., 2012). Traditional Chinese herbal treatment have been commonly used to treat MI for hundreds of years and recently, it has also been reported to exert protective effect against myocardial I/R injury (Liu et al., 2013; Li et al., 2014; Zhao et al., 2016a).

One example of the traditional Chinese medications is SanHuang-Xie-Xin-Tang (SHXT), which consists of three herbs: RC, Scutellariae radix and Rhei rhizome. Liou et al. (2011) showed that pre-treatment with SHXT was associated with significantly lower arrhythmia scores, reduced mortality rates and reduced levels of cardiac enzymes such as creatine kinase (CK), lactate dehydrogenase (LDH) and troponin I in rats with myocardial I/R injury. Additionally, there was reduced infarct size and decreased apoptosis in myocytes, partly due to modulation of endothelial nitric oxide synthase (eNOS) and MAPK pathways. While it is commonly used as a component of herbal mixtures, the extract of RC alone has been shown to possess cardioprotective effect against I/R damage. Administration of crude extract of RC for 7 days preceding acute myocardial injury in rats was associated with reduced myocardial injury as evidenced by decreased ST$\mathrm{T}$ elevation on electrocardiogram (ECG) and biochemically by decreased levels of CK and LDH. Other beneficial effects were reduction in oxidative stress reflected by reduced levels of malondiealdehyde (MDA) and increased activity of superoxide dismutase (SOD) (Liu et al., 2010).

Several active compounds identified in RC have been suggested as possible candidates accounting for the cardioprotective activities against I/R injury as demonstrated.
One of the compounds that appears to have cardioprotective activity is berberine. Although the cardioprotective activity of berberine is well recognized, the exact mechanism by which it protects from I/R injury remains unknown. Chang et al. (2012) found that pretreatment with berberine decreased infarct size and reduced the incidence as well as duration of arrhythmias in rats with I/R injury with demonstrable difference in AMPK activity in both non-ischemic areas (NIA) and myocardial areas at risk (AAR). Up-regulation of AMPK in the NIA helped to enhance cardiac function through increased uptake of glucose, glycolysis and oxidation of fatty acid whereas down-regulation of AMPK in the areas at risk helped to protect the cardiac myocytes from apoptosis. This protective effect of berberine was later specifically demonstrated in the CVD prone diabetic heart, as demonstrated by the regulation of myocardial energy metabolism by berberine during I/R injury (Chang et al., 2016). In myocardial $\mathrm{I} / \mathrm{R}$, there is always excessive autophagy which will lead to expansive degradation of cytosolic proteins and organelles, resulting in collapse of cellular functions. Huang et al. (2015) have reported the beneficial effect of berberine in lowering the cellular autophagy level in berberine-treated myocytes exposed to $I / R$ injury which were found to have reduced expression of autophagy-related proteins, which then led to suppression of autophagy activation. Berberine's cardioprotective action may also be associated with its antiapoptotic action. Based on both in vitro and in vivo results, berberine decreases I/R-induced myocardial apoptosis, as a result of modulation of the Notch1/ Hairy and enhancer of split 1(Hes1)- Phosphatase and tensin homolog deleted on chromosome 10 (PTEN)/Akt signaling pathway (Yu et al., 2015). In a diabetic rat model, berberine exerted anti-apoptotic activity through activation of AMPK and PI3K-Akt-eNOS signaling (Chen et al., 2014).

Mitochondrial dysfunction is an additional factor believed to be responsible for myocardial $\mathrm{I} / \mathrm{R}$ injury. Treatment with berberine has been shown to improve mitochondrial dysfunction, as demonstrated by improved mitochondrial membrane potential, increased mitochondrial complex I activity and reduced release of cytochrome $C$ from the mitochondria (Wang et al., 2015). Endoplasmic reticulum (ER) stress is also known to play an important part during $I / R$ injury. Unfolded protein response (UPR) is initiated in the myocardium after I/R and the continuous UPR causes upregulation of proapoptotic proteins. Consequently, cellular apoptosis occurs, resulting in myocardial I/R injury (Wu et al., 2014b). The role of berberine in modulation of ER stress level during myocardial I/R injury has also been described. Pretreatment with berberine has resulted in suppressed myocardial $I / R$ induced ER stress due to the activation of Janus kinase 2/signal transducer and activator of transcription 3 (JAK2/STAT3) signaling (Zhao et al., 2016b) An additional mechanism by which berberine is able to ameliorate myocardial I/R injury is via repression of the inflammatory response. Lower levels of inflammatory markers, namely the cytokines tumor necrosis factor (TNF)- $\alpha$, IL- 6 and IL- $\beta$ were found in rats pre-treated with berberine prior to induction of myocardial ischemia; this was believed to be mediated by downregulation of PI3K/AKT 
signaling thus preventing myocardial $\mathrm{I} / \mathrm{R}$ injury (Zhu and $\mathrm{Li}$, 2016).

Coptisine is one of the other compounds in RC that might have a role in prevention of $I / R$ injury. In a rat model, administration of coptisine alleviated I/R-induced arrhythmias and attenuated the reduction in ejection fraction as well as fractional shortening on echocardiography. Treatment with coptisine was also associated with reduced infarct size, suppressed myocardial apoptosis and reduced proinflammatory cytokines. Based on the reduced expression of Rho, Rho-kinase 1 (ROCK1), ROCK2 and attenuation of myosin phosphatase targeting subunit-1 phosphorylation, it was speculated that inhibition of the Rho/ROCK pathway was likely to be coptisine's mechanism of cardioprotection (Guo et al., 2013).

Palmatine, another component contained in RC extract, is also recognized as a potential cardioprotective agent. The administration of palmatine to rats prior to myocardial $\mathrm{I} / \mathrm{R}$ injury was associated with reduced I/R-induced myocardial dysfunction as evidenced by inhibition of the expected increase in $\mathrm{LDH}, \mathrm{CK}$, and MDA, as well as significant reduction in cyclooxygenase-2 (COX-2) and inducible nitric oxide synthase (iNOS) expression. Interestingly, in vitro studies using human aortic endothelial cells resulted in increased heme-oxygenase1 induction, indicating the strong antioxidant and antiinflammatory action of palmatine (Kim et al., 2009). Based on the studies, RC is a potential protective agent against myocardial $\mathrm{I} / \mathrm{R}$ injury through its active compounds which exert different protection actions including regulation of cellular energy metabolism, anti-apoptosis effect, protection against mitochondrial dysfunction and ER stress as well as antioxidant and anti-inflammatory action.

\section{CONCLUSION}

In conclusion, results of recent studies appear to support for the usage of RC for treatment of CVDs and related conditions. They have provided scientific evidence that RC's use in traditional Chinese medicine for the last 2000 years was likely to have truly resulted in desirable effects on CVDs via its effects on

\section{REFERENCES}

Ahn, T. G., Lee, J. Y., Cheon, S. Y., An, H. J., and Kook, Y. B. (2013). Protective effect of Sam-Hwang-Sa-Sim-Tang against hepatic steatosis in mice fed a highcholesterol diet. BMC Complement. Altern. Med. 13:366. doi: 10.1186/14726882-13-366

Cao, J., Han, Z., Tian, L., Chen, K., Fan, Y., Ye, B., et al. (2014). Curcumin inhibits EMMPRIN and MMP-9 expression through AMPK-MAPK and PKC signaling in PMA induced macrophages. J. Transl. Med. 12:266. doi: 10.1186/s12967-0140266-2

Cao, Y., Bei, W., Hu, Y., Cao, L., Huang, L., Wang, L., et al. (2012). Hypocholesterolemia of Rhizoma Coptidis alkaloids is related to the bile acid by up-regulated CYP7A1 in hyperlipidemic rats. Phytomedicine 19, 686-692. doi: 10.1016/j.phymed.2012.03.011

Chan, C. K., Goh, B. H., Kamarudin, M. N. A., and Kadir, H. A. (2012). Aqueous fraction of Nephelium ramboutan-ake rind induces mitochondrial-mediated apoptosis in HT-29 human colorectal adenocarcinoma cells. Molecules 17, 6633-6657. doi: 10.3390/molecules 17066633 the various risk factors and biochemical pathways involved in CVDs pathogenesis. RC appears to have tremendous potential as a cardioprotective agent given its ability to improve a large number of parameters associated with increased risk of CVDs including anti-atherosclerotic effect, lipid-lowering effect, anti-obesity effect and anti-hepatic steatosis effect. Aside from reducing incidence, it also reduces the damage caused by CVDs as it confers protection from myocardial I/R injury. These properties are mainly attributed to its bioactive compounds: berberine, coptisine, palmatine, epiberberine, jatrorrhizine and magnoflorine. However, knowledge regarding the underlying mechanisms of action of these compounds is still limited. More research investigating the exact mechanisms of RC's cardioprotective activities is needed. This is to fully exploit this traditional remedy's potential to contribute to the development of new cardioprotective agents as this could be a new opportunity to reduce global prevalence of CVDs.

\section{AUTHOR CONTRIBUTIONS}

HT and BG contributed to the literature database search, data collection, data extraction, and writing of the manuscript. PP, SS, $\mathrm{AD}, \mathrm{TM}, \mathrm{KC}, \mathrm{LL}$, and $\mathrm{BG}$ contributed vital insight and proofread on the writing. The research topic was conceptualized by BG.

\section{ACKNOWLEDGMENTS}

This work was inspired by Monash Pharmacy Degree Course, Unit PAC2412 which entitled "Integrated therapeutics: Introduction and cardiovascular" and financially supported by the MONASH PVC Award Grant (Project Q7 No. PVC-ECR-2016), Monash University Malaysia ECR Grant (5140077-000-00), External Industry Grant (Biotek Abadi Vote No. GBA-808813), Fundamental Research Grant Scheme (FRGS/1/2014/SKK01/MUSM/03/2) of Malaysia Ministry of Higher Education, MOSTI eScience Funds (02-02-10-SF0215 and 06-02-10-SF0300), University of Malaya for High Impact Research Grant (UM-MOHE HIR Nature Microbiome Grant No. H-50001-A000027 and No. A000001-50001).

Chan, C. K., Supriady, H., Goh, B. H., and Kadir, H. A. (2015). Elephantopus scaber induces apoptosis through ROS-dependent mitochondrial signaling pathway in HCT116 human colorectal carcinoma cells. J. Ethnopharm. 168, 291-304. doi: 10.1016/j.jep.2015.03.072

Chang, W., Li, K., Guan, F., Yao, F., Yu, Y., Zhang, M., et al. (2016). Berberine pretreatment confers cardioprotection against ischemia-reperfusion injury in a rat model of type 2 diabetes. J. Cardiovasc. Pharmacol. Ther. 21, 486-494. doi: $10.1177 / 1074248415627873$

Chang, W., Zhang, M., Li, J., Meng, Z., Xiao, D., Wei, S., et al. (2012). Berberine attenuates ischemia-reperfusion injury via regulation of adenosine$5^{\prime}$-monophosphate kinase activity in both non-ischemic and ischemic areas of the rat heart. Cardiovasc. Drugs Ther. 26, 467-478. doi: 10.1007/s10557-012$6422-0$

Chen, B., Xue, D. F., Han, B., Kou, S. M., Ye, X. L., and Li, X. G. (2015). Regulatory effect of coptisine on key genes involved in cholesterol metabolism. Zhongguo Zhong Yao Za Zhi 40, 1548-1553. doi: 10.4268/cjcmm20150823

Chen, J., Zhao, H., Wang, X., Lee, F. S.-C., Yang, H., and Zheng, L. (2008). Analysis of major alkaloids in Rhizoma coptidis by capillary 
electrophoresis-electrospray-time of flight mass spectrometry with different background electrolytes. Electrophoresis 29, 2135-2147. doi: 10.1002/elps.200700797

Chen, K., Li, G., Geng, F., Zhang, Z., Li, J., Yang, M., et al. (2014). Berberine reduces ischemia/reperfusion-induced myocardial apoptosis via activating AMPK and PI3K-Akt signaling in diabetic rats. Apoptosis 19, 946-957. doi: 10.1007/s10495-014-0977-0

Chen, X. (2009). Medical Composition Containing Dioscorea Opposite, Coptis Chinensis, Vitamins, Major Elements and Trace Elements for Lowering Blood Sugar and Blood Lipid, and Diabetes Mellitus, and Its Formulation. CN. Patent No 101579464. Beijing: State Intellectual Property Office of the P.R.C.

Chi, L., Peng, L., Pan, N., Hu, X., and Zhang, Y. (2014). The anti-atherogenic effects of berberine on foam cell formation are mediated through the upregulation of sirtuin 1. Int. J. Mol. Med. 34, 1087-1093. doi: 10.3892/ijmm.2014.1868

Choi, J. S., Kim, J. H., Ali, M. Y., Min, B. S., Kim, G. D., and Jung, H. A. (2014). Coptis chinensis alkaloids exert anti-adipogenic activity on 3T3-L1 adipocytes by downregulating C/EBP- $\alpha$ and PPAR- $\gamma$. Fitoterapia 98, 199-208. doi: 10.1016/j.fitote.2014.08.006

Fan, H., Chen, Y. Y., Bei, W. J., Wang, L. Y., Chen, B. T., and Guo, J. (2013). In vitro screening for antihepatic steatosis active components within Coptidis Rhizoma Alkaloids Extract using liver cell extraction with HPLC analysis and a free fatty acid-induced hepatic steatosis HepG2 cell assay. Evid. Based Complement. Alternat. Med. 2013:459390. doi: 10.1155/2013/459390

Fan, H., Yang, L., Fu, F., Xu, H., Meng, Q., Zhu, H., et al. (2012). Cardioprotective effects of salvianolic acid A on myocardial ischemia-reperfusion injury in vivo and in vitro. Evid. Based Complement. Alternat. Med. 2012:508938. doi: $10.1155 / 2012 / 508938$

Frostegård, J. (2013). Immunity, atherosclerosis and cardiovascular disease. BMC Med. 11:117. doi: 10.1186/1741-7015-11-117

Guo, J. (2012). Chinese Medicinal Composition Containing Saponins of Panax and Alkaloids of Coptidis for Preventing and Treating Lipid Metabolism Disorder. WO. Patent No 2012100440. Geneva: World Intellectual Property Organization.

Guo, J., Wang, S. B., Yuan, T. Y., Wu, Y. J., Yan, Y., Li, L., et al. (2013). Coptisine protects rat heart against myocardial ischemia/reperfusion injury by suppressing myocardial apoptosis and inflammation. Atherosclerosis 231, 384-391. doi: 10.1016/j.atherosclerosis.2013.10.003

Guo, T., Woo, S. L., Guo, X., Li, H., Zheng, J., Botchlett, R., et al. (2016). Berberine ameliorates hepatic steatosis and suppresses liver and adipose tissue inflammation in mice with diet-induced obesity. Sci. Rep. 6:22612. doi: $10.1038 /$ srep22612

He, K., Hu, Y., Ma, H., Zou, Z., Xiao, Y., Yang, Y., et al. (2016). Rhizoma coptidis alkaloids alleviate hyperlipidemia in B6 mice by modulating gut microbiota and bile acid pathways. BBA Mol. Basis Dis. 1862, 1696-1709. doi: 10.1016/j.bbadis.2016.06.006

He, K., Kou, S., Zou, Z., Hu, Y., Feng, M., Han, B., et al. (2015a). Hypolipidemic effects of alkaloids from Rhizoma coptidis in diet-induced hyperlipidemic hamsters. Planta Med. 82, 690-697. doi: 10.1055/s-0035-1568261

He, K., Ye, X., Wu, H., Wang, Y., Zou, Z., Ning, N., et al. (2015b). The safety and anti-hypercholesterolemic effect of coptisine in Syrian golden hamsters. Lipids 50, 185-194. doi: 10.1007/s11745-014-3983-7

Ho, F. M., Liao, Y. H., Yang, A. J., Lee Chao, P. D., Hou, Y. C., Huang, C. T., et al. (2012). Anti-atherosclerotic action of Ger-Gen-Chyn-Lian-Tang and AMPK-dependent lipid lowering effect in hepatocytes. J. Ethnopharmacol. 142, 175-187. doi: 10.1016/j.jep.2012.04.034

Hsieh, Y. S., Kuo, W. H., Lin, T. W., Chang, H. R., Lin, T. H., Chen, P. N., et al. (2007). Protective effects of berberine against low-density lipoprotein (LDL) oxidation and oxidized LDL-induced cytotoxicity on endothelial cells. J. Agric. Food Chem. 55, 10437-10445. doi: 10.1021/jf071868c

Huang, C., Zhang, Y., Gong, Z., Sheng, X., Li, Z., Zhang, W., et al. (2006). Berberine inhibits 3T3-L1 adipocyte differentiation through the PPAR $\gamma$ pathway. Biochem. Biophys. Res. Commun. 348, 571-578. doi: 10.1016/j.bbrc.2006.07.095

Huang, Z., Han, Z., Ye, B., Dai, Z., Shan, P., Lu, Z., et al. (2015). Berberine alleviates cardiac ischemia/reperfusion injury by inhibiting excessive autophagy in cardiomyocytes. Eur. J. Pharmacol. 762, 1-10. doi: 10.1016/j.ejphar.2015.05.028

Huang, Z., Wang, L., Meng, S., Wang, Y., Chen, T., and Wang, C. (2011). Berberine reduces both MMP-9 and EMMPRIN expression through prevention of p38 pathway activation in PMA-induced macrophages. Int. J. Cardiol. 146, 153-158. doi: 10.1016/j.ijcard.2009.06.023

Huang, Z., Xu, X., Lu, F., Wang, N., Chen, G., Zhao, Y., et al. (2013). Jiao tai wan attenuates hepatic lipid accumulation in type 2 diabetes mellitus. Evid. Based Complement. Alternat. Med. 2013:567045. doi: 10.1155/2013/567045

Hung, T. M., Lee, J. P., Min, B. S., Choi, J. S., Na, M., Zhang, X., et al. (2007a). Magnoflorine from Coptidis Rhizoma protects high density lipoprotein during oxidant stress. Biol. Pharm. Bull. 30, 1157-1160. doi: 10.1248/bpb.30.1157

Hung, T. M., Na, M., Min, B. S., Zhang, X., Lee, I., Ngoc, T. M., et al. (2007b). Protective effect of magnoflorine isolated from coptidis rhizoma on $\mathrm{Cu} 2+$-induced oxidation of human low density lipoprotein. Planta Med. 73, 1281-1284. doi: 10.1055/s-2007-981615

Itabe, H. (2009). Oxidative modification of LDL: its pathological role in atherosclerosis. Clin. Rev. Allergy Immunol. 37, 4-11. doi: 10.1007/s12016-008$8095-9$

Kim, Y. M., Ha, Y. M., Jin, Y. C., Shi, L. Y., Lee, Y. S., Kim, H. J., et al. (2009). Palmatine from Coptidis rhizoma reduces ischemia-reperfusion-mediated acute myocardial injury in the rat. Food Chem. Toxicol. 47, 2097-2102. doi: 10.1016/j.fct.2009.05.031

Kou, S., Han, B., Wang, Y., Huang, T., He, K., Han, Y., et al. (2016). Synergetic cholesterol-lowering effects of main alkaloids from Rhizoma Coptidis in HepG2 cells and hypercholesterolemia hamsters. Life Sci. 151, 50-60. doi: 10.1016/j.lfs.2016.02.046

Kwak, D. H., Lee, J. H., Kim, D. G., Kim, T., Lee, K. J., and Ma, J. Y. (2013). Inhibitory effects of Hwangryunhaedok-tang in 3T3-L1 adipogenesis by regulation of Raf/MEK1/ERK1/2 Pathway and PDK1/Akt Phosphorylation. Evid. Based Complement. Alternat. Med. 2013:413906. doi: $10.1155 / 2013 / 413906$

Lavie, C. J., Milani, R. V., and Ventura, H. O. (2009). Obesity and cardiovascular disease: risk factor, paradox, and impact of weight loss. J. Am. Coll. Cardiol. 53 , 1925-1932. doi: 10.1016/j.jacc.2008.12.068

Li, G., Li, Y., Sun, F., Dong, X., and Zhong, F. (2015). Chinese Medicine Composition for Reducing Blood Glucose and Lipid Protecting Blood Vesssel Endothelial, and Preparation Method Thereof. CN. Patent No 104257839. Beijing: State Intellectual Property Office of the P.R.C.

Li, J. C., Shen, X. F., and Meng, X. L. (2013). A traditional Chinese medicine JiuHuangLian (Rhizoma coptidis steamed with rice wine) reduces oxidative stress injury in type 2 diabetic rats. Food Chem. Toxicol. 59, 222-229. doi: 10.1016/j.fct.2013.06.005

Li, X., Liu, J., Lin, L., Guo, Y., Lin, C., Zhang, C., et al. (2014). Traditional Chinese medicine Shuang Shen Ning Xin attenuates myocardial ischemia/reperfusion injury by preserving of mitochondrial function. Evid. Based Complement. Alternat. Med. 2014:180965. doi: 10.1155/2014/180965

Liou, S. F., Ke, H. J., Hsu, J. H., Liang, J. C., Lin, H. H., Chen, I. J., et al. (2011). SanHuang-Xie-Xin-Tang prevents rat hearts from ischemia/reperfusion-induced apoptosis through eNOS and MAPK Pathways. Evid. Based Complement. Alternat. Med. 2011:915051. doi: 10.1093/ecam/neq061

Liu, H., and Lu, H. Y. (2014). Nonalcoholic fatty liver disease and cardiovascular disease. World J. Gastroenterol. 20, 8407-8415. doi: 10.3748/wjg.v20.i26.8407

Liu, Q., Li, J., Wang, J., Li, J., Janicki, J. S., and Fan, D. (2013). Effects and mechanisms of Chinese herbal medicine in ameliorating myocardial ischemiareperfusion injury. Evid. Based Complement. Alternat. Med. 2013:925625. doi: $10.1155 / 2013 / 925625$

Liu, X., Chen, X., and Jin, R. (2010). Protective effects of Rhizoma coptidis on acute myocardial ischemia injured cardiomyocytes in vivo and in vitro. Chin. J. Info. Tradit. Chin. Med. 17, 28-30. doi: 10.3969/j.issn.1005-5304.2010.12.012

Lucas, A. D., and Greaves, D. R. (2001). Atherosclerosis: role of chemokines and macrophages. Expert Rev. Mol. Med. 3, 1-18. doi: 10.1017/S1462399401003696

Ma, B. L., Yao, M. K., Zhong, J., Ma, Y. M., Gao, C. L., Wu, J. S., et al. (2012). Increased systemic exposure to rhizoma coptidis alkaloids in lipopolysaccharide-pretreated rats attributable to enhanced intestinal absorption. Drug Metab. Dispos. 40, 381-388. doi: 10.1124/dmd.111. 041152

Ma, H., Hu, Y., Zou, Z., Feng, M., Ye, X., and Li, X. (2016). Antihyperglycemia and antihyperlipidemia effect of protoberberine alkaloids from Rhizoma Coptidis in HepG2 cell and diabetic KK-Ay mice. Drug Dev. Res. 77, 163-170. doi: $10.1002 / \mathrm{ddr} .21302$ 
Moghadamtousi, S., Goh, B., Chan, C., Shabab, T., and Kadir, H. (2013). Biological activities and phytochemicals of swietenia macrophylla king. Molecules 18, 10465-10483. doi: 10.3390/molecules 180910465

Moukdar, F., Robidoux, J., Lyght, O., Pi, J., Daniel, K. W., and Collins, S. (2009). Reduced antioxidant capacity and diet-induced atherosclerosis in uncoupling protein-2-deficient mice. J. Lipid Res. 50, 59-70. doi: 10.1194/jlr.M800273JLR200

Ning, N., He, K., Wang, Y., Zou, Z., Wu, H., Li, X., et al. (2015). Hypolipidemic effect and mechanism of palmatine from Coptis chinensis in hamsters fed high-fat diet. Phytother. Res. 29, 668-673. doi: 10.1002/ ptr.5295

Pisto, P., Santaniemi, M., Bloigu, R., Ukkola, O., and Kesäniemi, Y. A. (2014). Fatty liver predicts the risk for cardiovascular events in middle-aged population: a population-based cohort study. BMJ Open 4:e004973. doi: 10.1136/bmjopen2014-004973

Remppis, A., Bea, F., Greten, H. J., Buttler, A., Wang, H., Zhou, Q., et al. (2010). Rhizoma coptidis inhibits LPS-induced MCP-1/CCL2 production in murine macrophages via an AP-1 and NFKB-dependent pathway. Mediators Inflamm. 2010:194896. doi: 10.1155/2010/194896

Supriady, H., Kamarudin, M. N. A., Chan, C. K., Goh, B. H., and Kadir, H. A. (2015). SMEAF attenuates the production of pro-inflammatory mediators through the inactivation of Akt-dependent NF-KB, p38 and ERK1/2 pathways in LPS-stimulated BV-2 microglial cells. J. Funct. Food. 17, 434-448. doi: 10.1016/j.jff.2015.05.042

Tan, H. L., Chan, K. G., Pusparajah, P., Lee, L. H., and Goh, B. H. (2016). Gynura procumbens: an overview of the biological activities. Front. Pharmacol. 7:52. doi: 10.3389/fphar.2016.00052

Tan, L. T. H., Lee, L. H., Yin, W. F., Chan, C. K., Kadir, H. A., Chan, K. G., et al. (2015). Traditional uses, phytochemistry, and bioactivities of Cananga odorata (Ylang-Ylang). Evid. Based Complement Alternat Med. 2015:896314. doi: $10.1155 / 2015 / 896314$

Tang, L. Q., Wei, W., Chen, L. M., and Liu, S. (2006). Effects of berberine on diabetes induced by alloxan and a high-fat/high-cholesterol diet in rats. J. Ethnopharmacol. 108, 109-115. doi: 10.1016/j.jep.2006.04.019

Trapani, L., Segatto, M., and Pallottini, V. (2012). Regulation and deregulation of cholesterol homeostasis: the liver as a metabolic "power station." World J. Hepatol. 4, 184-190. doi: 10.4254/wjh.v4.i6.184

Vijayaraghavan, K. (2010). Treatment of dyslipidemia in patients with type 2 diabetes. Lipids Health Dis. 9, 1-12. doi: 10.1186/1476-511X-9-144

Walden, R., and Tomlinson, B. (2011). "Cardiovascular disease," in Herbal Medicine: Biomolecular and Clinical Aspects, eds I. F. F. Benzie and S. WachtelGalor (Boca Raton, FL: LLC), 333-361.

Wallace, T. C. (2011). Anthocyanins in cardiovascular disease. Adv. Nutr Int. Rev. J. 2, 1-7. doi: 10.3945/an.110.000042

Wang, H., Mu, W., Shang, H., Lin, J., and Lei, X. (2014). The antihyperglycemic effects of Rhizoma Coptidis and mechanism of actions: a review of systematic reviews and pharmacological research. Biomed. Res. Int. 2014:10. doi: $10.1155 / 2014 / 798093$

Wang, Q., Zhang, M., Liang, B., Shirwany, N., Zhu, Y., and Zou, M. H. (2011). Activation of AMP-activated protein kinase is required for berberine-induced reduction of atherosclerosis in mice: the role of uncoupling protein 2. PLoS ONE 6:e25436. doi: 10.1371/journal.pone.0025436

Wang, X. (2015). A Chinese Medicine Composition for Treating Obesity Complicated with Hyperlipidemia and Its Application. CN. Patent No 104645055. Beijing: State Intellectual Property Office of the P.R.C.

Wang, Y., Liu, J., Ma, A., and Chen, Y. (2015). Cardioprotective effect of berberine against myocardial ischemia/reperfusion injury via attenuating mitochondrial dysfunction and apoptosis. Int. J. Clin. Exp. Med. 8:14513.

Wu, H., He, K., Wang, Y., Xue, D., Ning, N., Zou, Z., et al. (2014a). The antihypercholesterolemic effect of jatrorrhizine isolated from Rhizoma Coptidis. Phytomedicine 21, 1373-1381. doi: 10.1016/j.phymed.2014. 05.002

Wu, H., Tang, Q., Yang, J., Ding, J., Ye, M., and Dong, W. (2014b). Atorvastatin ameliorates myocardial ischemia/reperfusion injury through attenuation of endoplasmic reticulum stress-induced apoptosis. Int. J. Clin. Exp. Med. 7, 4915-4923.
Wu, H., Wang, Y. Z., Wang, D. Z., Pang, J., Ye, X. L., and Li, X. G. (2014c). Effects of alkaloids from coptidis rhizoma on blood lipid metabolism and low-denstity lipoprotein receptor mRNA in golden hamsters. Zhongguo Zhong Yao Za Zhi 39, 2102-2105. doi: 10.4268/cjcmm20141131

Wu, J., Zhang, H., Hu, B., Yang, L., Wang, P., Wang, F., et al. (2016). Coptisine from Coptis chinensis inhibits production of inflammatory mediators in lipopolysaccharide-stimulated RAW 264.7 murine macrophage cells. Eur. J. Pharmacol. 780, 106-114. doi: 10.1016/j.ejphar.2016.03.037

Xiao, J., Fai So, K., Liong, E. C., and Tipoe, G. L. (2013). Recent advances in the herbal treatment of non-alcoholic Fatty liver disease. J. Tradit. Complement. Med. 3, 88-94. doi: 10.4103/2225-4110.110411

Xie, W., Gu, D., Li, J., Cui, K., and Zhang, Y. (2011). Effects and action mechanisms of berberine and Rhizoma coptidis on gut microbes and obesity in highfat diet-fed C57BL/6J mice. PLoS ONE 6:e24520. doi: 10.1371/journal.pone. 0024520

Ye, X., Li, X., and Yuan, L. (2009). Hypolipidemia Composite Preparation Comprising Rhizoma Coptidis Alkaloid and Crataegus Pinnatifida Flavanoid. CN. Patent No 10485752. Beijing: State Intellectual Property Office of the P.R.C.

Yi, J., Ye, X., Wang, D., He, K., Yang, Y., Liu, X., et al. (2013). Safety evaluation of main alkaloids from Rhizoma Coptidis. J. Ethnopharmacol. 145, 303-310. doi: 10.1016/j.jep.2012.10.062

Yokozawa, T., Ishida, A., Cho, E. J., and Nakagawa, T. (2003). The effects of Coptidis Rhizoma extract on a hypercholesterolemic animal model. Phytomedicine 10, 17-22. doi: 10.1078/094471103321648610

Yu, L., Li, F., Zhao, G., Yang, Y., Jin, Z., Zhai, M., et al. (2015). Protective effect of berberine against myocardial ischemia reperfusion injury: role of Notch1/Hes1PTEN/Akt signaling. Apoptosis 20, 796-810. doi: 10.1007/s10495-015-1122-4

Yuan, X., Wang, J., Tang, X., Li, Y., Xia, P., and Gao, X. (2015). Berberine ameliorates nonalcoholic fatty liver disease by a global modulation of hepatic mRNA and lncRNA expression profiles. J. Transl. Med. 13:24. doi: 10.1186/s12967-015-0383-6

Zhang, Z., Li, B., Meng, X., Yao, S., Jin, L., Yang, J., et al. (2016). Berberine prevents progression from hepatic steatosis to steatohepatitis and fibrosis by reducing endoplasmic reticulum stress. Sci. Rep. 6:20848. doi: 10.1038/srep20848

Zhao, G. L., Yu, L. M., Gao, W. L., Duan, W. X., Jiang, B., Liu, X. D., et al. (2016b). Berberine protects rat heart from ischemia/reperfusion injury via activating JAK2/STAT3 signaling and attenuating endoplasmic reticulum stress. Acta Pharmacol. Sin. 37, 354-367. doi: 10.1038/aps.2015.136

Zhao, Y., Xu, L., Qiao, Z., Gao, L., Ding, S., Ying, X., et al. (2016a). YiXin-Shu, a ShengMai-San-based traditional Chinese medicine formula, attenuates myocardial ischemia/reperfusion injury by suppressing mitochondrial mediated apoptosis and upregulating liver-X-receptor $\alpha$. Sci. Rep. 6:23025. doi: $10.1038 /$ srep23025

Zhou, Y., Cao, S., Wang, Y., Xu, P., Yan, J., Bin, W., et al. (2014). Berberine metabolites could induce low density lipoprotein receptor up-regulation to exert lipid-lowering effects in human hepatoma cells. Fitoterapia 92, 230-237. doi: 10.1016/j.fitote.2013.11.010

Zhu, Q. W., and Li, Y. G. (2016). Berberine attenuates myocardial ischemia reperfusion injury by suppressing the activation of PI3K/AKT signaling. Exp. Ther. Med. 11, 978-984. doi: 10.3892/etm.2016.3018

Zou, Z. Y., Hu, Y. R., Ma, H., Feng, M., Li, X. G., and Ye, X. L. (2016). Epiberberine reduces serum cholesterol in diet-induced dyslipidemia Syrian golden hamsters via network pathways involving cholesterol metabolism. Eur. J. Pharmacol. 774, 1-9. doi: 10.1016/j.ejphar.2015.11.017

Conflict of Interest Statement: The authors declare that the research was conducted in the absence of any commercial or financial relationships that could be construed as a potential conflict of interest.

Copyright () 2016 Tan, Chan, Pusparajah, Duangjai, Saokaew, Mehmood Khan, Lee and Goh. This is an open-access article distributed under the terms of the Creative Commons Attribution License (CC BY). The use, distribution or reproduction in other forums is permitted, provided the original author(s) or licensor are credited and that the original publication in this journal is cited, in accordance with accepted academic practice. No use, distribution or reproduction is permitted which does not comply with these terms. 